\title{
A Patient with Stage IVB Cervical Cancer with Para-aortic Lymph Node (PAN) and Supraclavicular Lymph Node (SCN) Metastases Who Reached Long-term Survival with Chemoradiotherapy
}

\author{
Hiromichi Gomi ${ }^{1}$, Mio Shinozaki ${ }^{1}$, Yukinori Okada ${ }^{1}$, Tatsuyuki Abe ${ }^{1}$, \\ Yasuo Nakajima ${ }^{1}$, Tatsuru Ohara², Akiko Tozawa' ${ }^{2}$ and Nao Suzuki ${ }^{2}$
}

(Received for Publication: March 26, 2015)

\begin{abstract}
We report a case of a patient who demonstrated long-term survival following completion of concurrent chemoradiotherapy (CCRT) for stage IVB advanced uterine cervical cancer. When the patient was referred to us, her radiological findings showed para-aortic and supraclavicular lymph node (SCN) metastases. The CCRT for primary lesions and para-aortic lymph node (PAN) were performed, followed by radiotherapy for SCN, resulting in successful control of the lesions. After five and a half years of strict follow-up, recurrent PAN was observed with PET-CT. Systemic chemotherapy was re-stored and controlled recurrent PAN without signs of serious adverse events. The patient has showed no recurrent signs with any related symptoms for the past 8 years since the initial treatment. As seen in this case, favorable prognosis can be achieved even for stage IVB advanced uterine cervical cancer patients, when the distant metastases are located in lymph nodes where CCRT could fully cover.
\end{abstract}

\section{Key words}

uterine cervical cancer, supraclavicular lymph node metastasis, chemoradiotherapy, prognosis

\section{Introduction}

Recently, favorable results have become anticipated with concurrent chemoradiotherapy (CCRT) for locally-advanced cancer in which the lesion is confined to the interior of the pelvis, even if the cervical cancer is an advanced case ${ }^{1-3)}$. However, the prognosis is still unfavorable for stage IVB cases in which distant metastases exist. Compared to hematogenous metastases, metastases localized in the lymph nodes are said to have a relatively favorable prognosis ${ }^{4}$. And among lymph node metastases, cases of metastases localized to the para-aortic lymph node (PAN) have become increasingly curable. A major reason is that extended field radiotherapy, including the pelvic and PAN areas, has become possi- ble due to advances in radiation therapy technology ${ }^{5)}$. However, the prognosis for metastases extending to the supraclavicular lymph node ( $\mathrm{SCN})$ remains unfavorable as distant metastases often manifest later. Here, we report the case of a patient who presented with PAN and SCN metastases in the diagnostic imaging at the initial visit and who had stage IVB cervical cancer but no other metastases. We performed chemoradiotherapy and the patient was able to achieve prolonged survival.

\section{Case Report}

A 56-year-old woman made a consultation with the chief complaint of genital bleeding for two years and mild abdominal pain. In the palpation at the initial visit, she had an induration $7 \mathrm{~cm}$ in diameter in

1 Department of Radiology, St. Marianna University School of Medicine

2 Department of Obstetrics and Gynecology, St. Marianna University School of Medicine 
the uterine cervix and bilateral parametrial infiltration. The infiltration reached the right pelvic wall. An induration of the lymph node $2 \mathrm{~cm}$ in diameter on the left supraclavicular fossa was palpable. We identified squamous cell carcinoma in the biopsy of the primary lesion (Fig. 1). In the diagnostic imaging with the MRI, a large primary tumor about $7 \mathrm{~cm}$ in diameter was detected in the uterine cervix (Fig. 2A). In the $\mathrm{CT}$, there were multiple enlarged lymph nodes about $1-2 \mathrm{~cm}$ in the minor axis in the pelvis, PAN area (Fig. 3A), and SCN (Fig. 4A). In the 2-[ $\left.{ }^{8} \mathrm{~F}\right]$ fluoro-2-deoxy-D-glucose positron emission tomography (FDGPET), markedly increased FDG uptake lesions were detected in the primary, pelvic lymph nodes (PN), the PAN, and the left SCN (Fig. 5). We did not identify any other abnormal accumulation. From the above, we determined that it was a case of stage IVB cervical squamous cell carcinoma, with metastases detected in the PAN and the SCN as extra pelvic lesions. While a pathological examination was implemented on the primary lesion, the PAN and SCN metastases were only diagnosed from the imaging. There was no anemia, and no abnormality in liver and renal function. The serum squamous cell carcinoma antigen (SCC) displayed an abnormally high $15.5 \mathrm{ng} / \mathrm{dl}$.
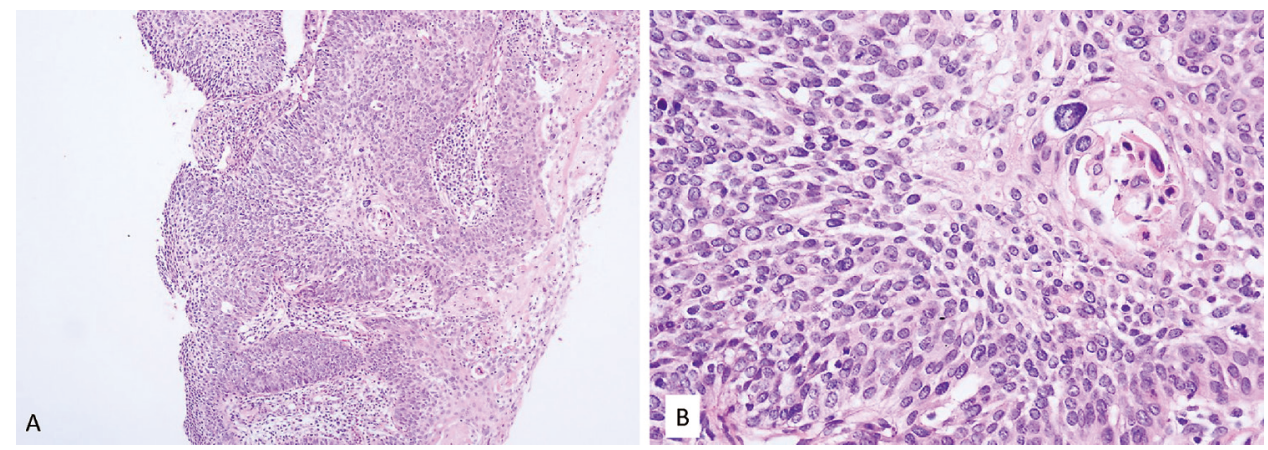

Fig. 1. Photomicrographs of cervical tumor biopsy specimen. (A) (original magnification x 100) Invasive squamous cell carcinoma. The tumor displays angular protrusions diagnostic of invasion, while the stroma of the invasive tumor displays a desmoplastic response. (B) (original magnification x 400) Invasive squamous cell carcinoma. The nuclei are enlarged and have keratinizing cells.
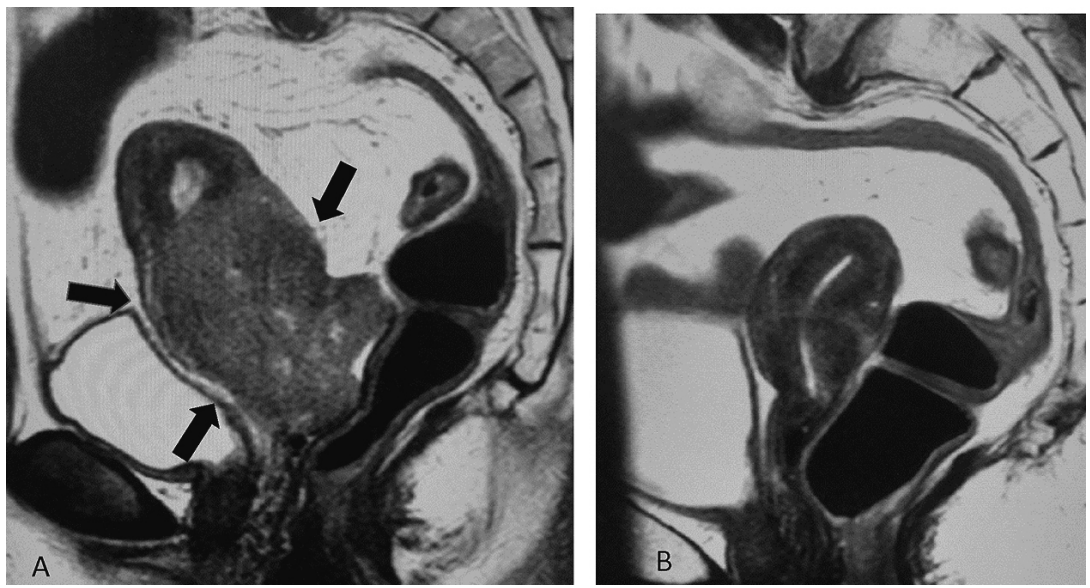

Fig. 2. (A) T2 weighted sagittal MRI before treatment shows a tumor (solid arrow) $7 \mathrm{~cm}$ in diameter in the uterine cervix. (B) T2 weighted sagittal MRI after treatment shows disappearance of large uterine tumor. 

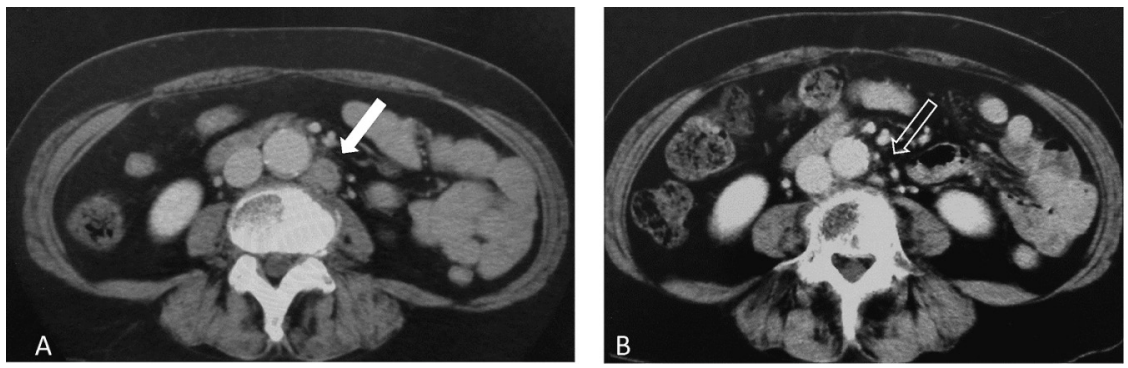

Fig. 3. (A) The contrast-enhanced CT before treatment at the L4 level shows enlarged lymph node (solid arrow) $2 \mathrm{~cm}$ in diameter in para-aortic area. (B) This finding disappears (open arrow) in the after-treatment CT.
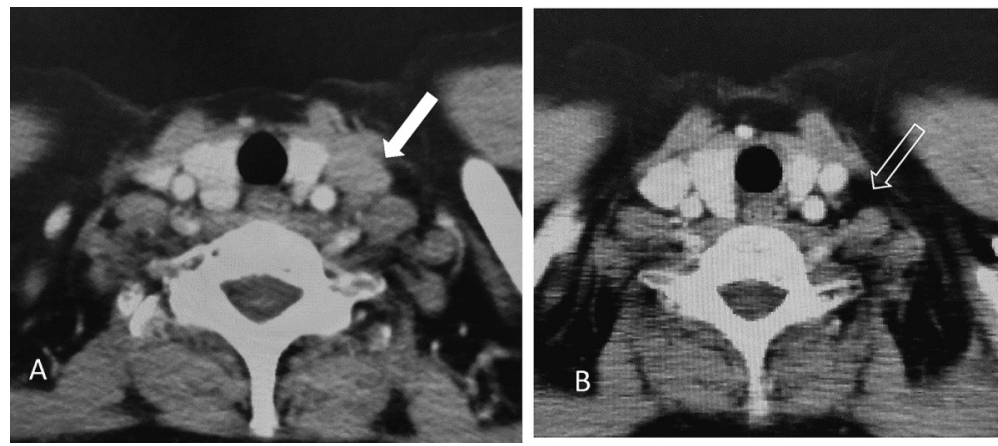

Fig. 4. (A) The pretreatment contrast-enhanced CT of neck shows enlarged lymph node (solid arrow) $2 \mathrm{~cm}$ in diameter on left supraclavicular fossa. (B) This finding disappears (open arrow) in the after-treatment CT.

\section{Treatment Progress}

The patient underwent concurrent chemoradiotherapy (CCRT) for the pelvis, the PAN, and the left $\mathrm{SCN}$. We performed external beam radiotherapy and intracavitary irradiation for the pelvis and the PAN radiation therapy. We began external beam radiotherapy with whole pelvic irradiation (Fig. 6A) using a $10 \mathrm{MV} \mathrm{X}$-ray of linear accelerator. After irradiating 30.6Gy/17f, we applied an extended irradiation field (pelvis + PAN area) (Fig. 6B), and then we placed the center splitter on the pelvis. After the pelvic total dose was complete, we focused and boosted the radiation field on the PAN area. The total external dose of the pelvis was 50.4Gy/28f. After irradiating $39.6 \mathrm{~Gy} / 22 \mathrm{f}$ in the PAN area, we further confined the radiation field for the tumefaction in the PAN we detected in the imaging and boosted it for a total dose of 50.4Gy. We began intracavitary irradiation once we inserted the center splitter for the external pelvic field. We irradiated a total dose of $12 \mathrm{~Gy}$ to point $\mathrm{A}$ using a high-dose-rate remote after loading system
(HDR-RALS) 3 times at one week intervals. With respect to chemotherapy, the patient received cisplatin (CDDP) $100 \mathrm{mg} /$ body and fluorouracil (5FU) $1000 \mathrm{mg} /$ body with one shot as an intra-arterial injection for the primary lesion. Although we began external beam radiotherapy one week later, we combined this with systemic administration of nedaplatin (NDP) $30 \mathrm{mg} / \mathrm{m}^{2} 8$ times during the external beam radiotherapy in one-week intervals. After the irradiation of the pelvis and PAN was complete, we irradiated the SCN (Fig. 7). After irradiating 40Gy/20f with a $6 \mathrm{MV} \mathrm{X}$ - ray beam, we reduced the radiation field for the metastatic lesion we detected in the imaging and boosted it to a total dose of 56Gy/28f. We were able to complete the chemoradiotherapy without any serious adverse event. After the radiation therapy, all lesions from the imaging and touch examination disappeared and we were able to gain control. The MRI (Fig. 2B) of the primary lesion, the CT scan of the PAN, and the SCN are shown in Fig. 3B and Fig. 4B, respectively. At the beginning of treat- 


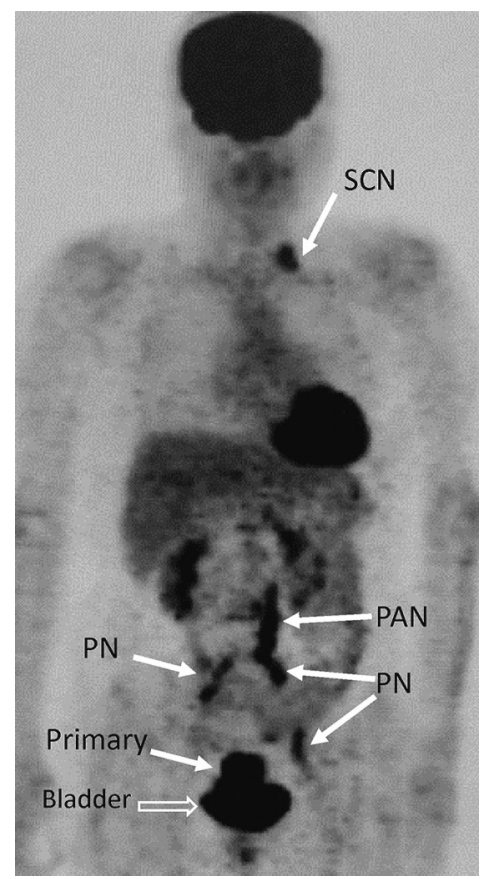

Fig. 5. The pretreatment FDG-PET image shows the primary tumor (solid arrow) of markedly increased FDG uptake, overlapping the bladder (open arrow), in the uterine cervix. There are multiple metastases involved the pelvic lymph nodes (PN), the para-aortic lymph nodes (PAN), and the left supraclavicular lymph nodes (SCN). (solid arrows)

ment the SCC was $15.5 \mathrm{ng} / \mathrm{dl}$; however, it decreased to $1.8 \mathrm{ng} / \mathrm{dl}$ when the pelvis and the PAN irradiation were completed, and when the SCN irradiation was complete it returned to normal limits at $0.8 \mathrm{ng} / \mathrm{dl}$. We continued follow-up observations at 1- to 2-month intervals thereafter and found no problems.

Five and a half years after the initial treatment, however, an abdominal CT scan showed a roundshaped mass about $1 \mathrm{~cm}$ in diameter in the para-aortic area at the L5 level. The serum level of SCC was within normal limits without any particular clinical symptoms. We implemented a strict follow-up by CT scan. The PET-CT scan 6 months later showed an increase in FDG uptake at the para-aortic site seen in the previous CT scan (Fig. 8A-C). Alhough the differential diagnosis included benign lymph node swel- ling and aortic aneurysm, the abnormal accumulation with the PET defied explanation. We considered an exploratory laparotomy biopsy, but the patient declined. We had no pathological proof and the SCC value was within normal limits, but we determined it to be a lymph node recurrence based on the findings of PET-CT. We implemented 6 courses of systemic chemotherapy with irinotecan hydrochloride hydrate (CPT11) and NDP. A CT scan was performed one month after completion of the chemotherapy, at which time the para-aortic mass had disappeared (Fig. 8D). At present, one year and six months have passed since the completion of the chemotherapy, marking almost 8 years since the initial treatment.

\section{Discussion}

Alhough the prognosis for stage IVB of cervical cancer is unfavorable, in contrast to hematogenous metastases, metastases confined to the lymph nodes are said to have a more favorable prognosis ${ }^{4}$. Treatment of cervical cancer lymph node metastasis progresses along with treatment for PN, PAN, and SCN; however, the prognosis is unfavorable with these advances. Kidd et al. have reported on this ${ }^{6)}$; their research examined lymph node metastasis staging by PET for pretreatment in relation to prognosis for 560 cervical cancer patients. There are a variety of treatment methods including surgery, surgery + postoperative irradiation, radiotherapy alone, and chemoradiotherapy, but this research does not analyze each method. According to this study, the two-year disease-specific survival rate (DSS) for the most advanced lymph node metastases is $80 \%$ for PN and $50 \%$ for PAN, but only $19 \%$ for SCN. When the stage of the primary lesion is limited to stage III, the twoyear DSS for the most advanced lymph node metastases is $60 \%$ for PN, $30 \%$ for PAN, and less than $10 \%$ for $\mathrm{SCN}$; there was no three-year survival. In the present case, the stage of the primary lesion was in the vicinity of stage III and had an estimated twoyear DSS of less than $10 \%$.

We show the reported outcomes for each specific form of SCN metastases treatment below. In the 2003 report by Tran et al. ${ }^{7)}$, the median survival of 7 cases treated with CCRT that included CDDP was 8 months. The ratio of $\mathrm{SCN}$ cases receiving irradiation was only 2 out of 7 , and the dose was $45 \mathrm{~Gy}$. In the 2007 report by Qiu et al. ${ }^{8}$, 24 out of 33 cases received CCRT, 9 subjects received radiotherapy alone, and the SCN was irradiated with 30-60Gy. The median length of survival was 15 months, and the three- 

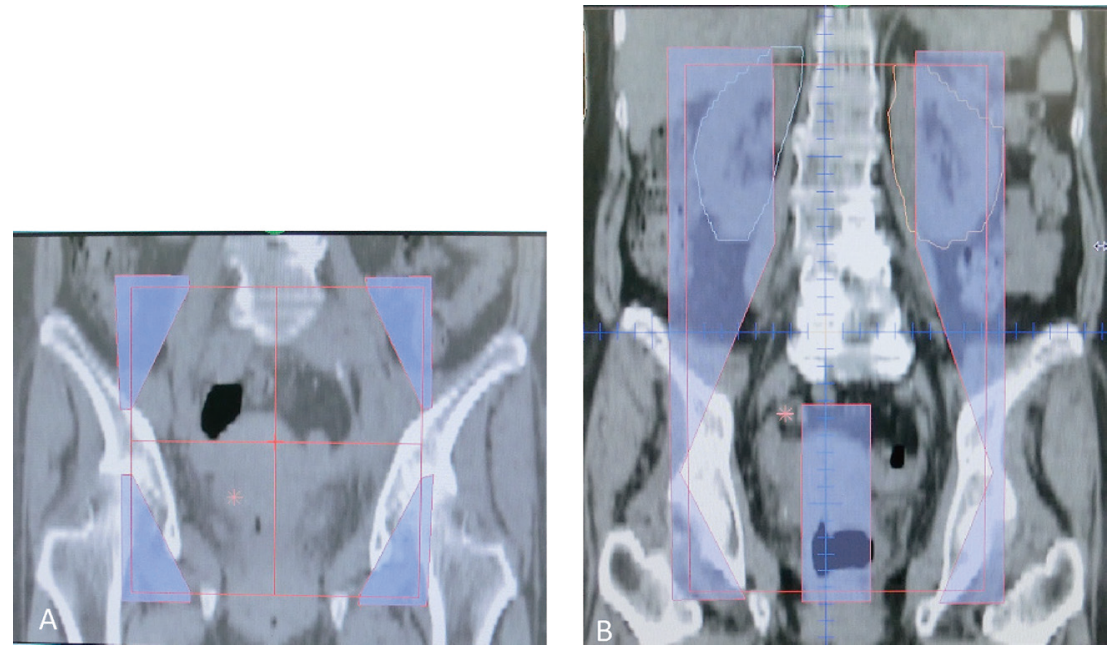

Fig. 6. (A) Whole pelvic irradiation field. (B) Extended irradiation field (pelvis + PAN area).

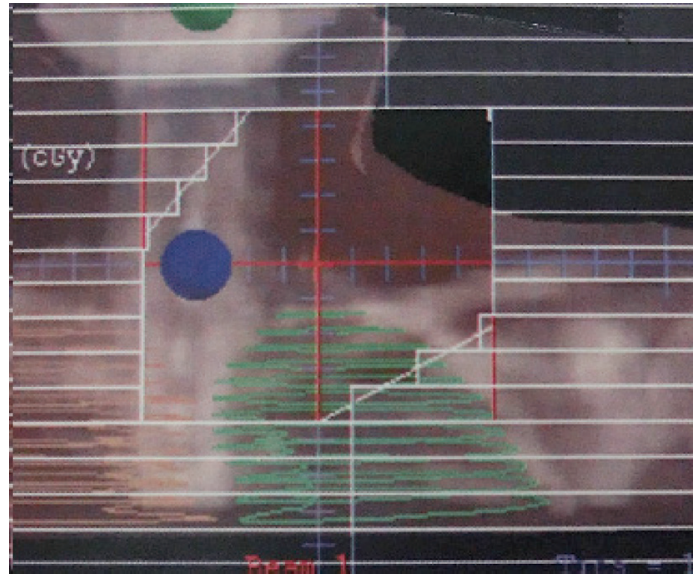

Fig. 7. Supraclavicular irradiation field. After irradiating $40 \mathrm{~Gy} / 20 \mathrm{f}$ with this field, we reduced the radiation field for the metastatic lesion we detected in the imaging and boosted it.

year over-all survival rate (OAS) was $16.5 \%$. In the 2008 report by Chao et al. ${ }^{9}$, all 12 of their cases received CCRT treatment and the SCN was irradiated with 30-60Gy. Their two-year OAS was $24.7 \%$. In the 2010 report by Kim K et al. ${ }^{4}$, 9 cases were treated with CCRT and the SCN was irradiated with 5070Gy. Three cases (33\%) survived disease-free for more than two years. In the 2012 report by Kim JK et al. ${ }^{10)}$, all 25 of their patients received CCRT and the SCN for all were irradiated with 59.4Gy. As a result, the three-year OAS was $49 \%$ and the three-year dis- ease-free survival rate (DFS) was 33\%. Thus, most have shown favorable outcomes. These results suggest that even among cases of cervical cancer with distant metastases, these metastases can be restricted to the lymph nodes as far as the SCN, and a curative dose can be irradiated to the interior of the pelvis and the metastatic lesion. In addition, if concurrent chemotherapy can be completed, an OAS of approximately $2-3$ years can be achieved for $20-30 \%$ of subjects, and considering the report by Kim JK, the OAS may exceed $40 \%$. We completed all curative radiotherapy and concurrent chemotherapy on the primary lesion and detected metastatic lesions for the patient in this report. Though the frequency is still low, we can say that this case fulfills the conditions for a complete cure.

Recurrence patterns of metastases reaching the SCN are assumed to have distant metastases that are generally outside the radiation field. All seven cases of SCN metastases cited above in Tran et al. ${ }^{7)}$ passed away, from distant metastasis after CCRT. In addition, as one might expect, among the cases in which metastases reached the SCN in Kim JK et al. ${ }^{10)}$, 15 of the 25 cases $(60 \%)$ that received CCRT were distant recurrences. Therefore, they were recommended frequent follow-up with PET-CT scans and adjuvant chemotherapy after CCRT had been completed. The case we are reporting did not have distant metastasis outside the radiation field; even though we have no pathological proof, there was a PAN recurrence inside the radiation field. We believe that implementing 

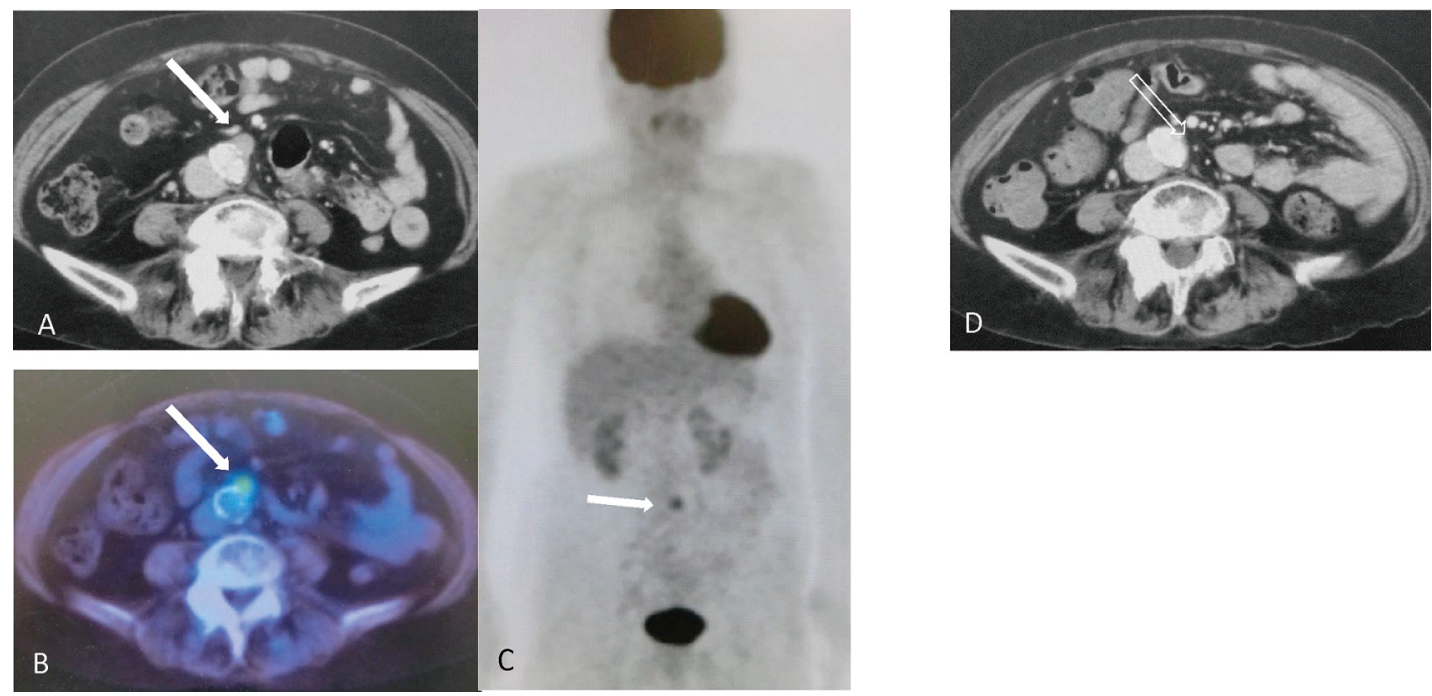

Fig. 8. (A) Five and a half years after the initial treatment, contrast-enhanced abdominal CT scan shows a round shaped mass about $1 \mathrm{~cm}$ in diameter attached to the abdominal aorta at the L5 level. (B)(C) The PET-CT scan 6 months later shows increased FDG uptake at this site (solid arrows). The patient underwent systemic chemotherapy. (D) One month after completion of the chemotherapy, soft tissue mass disappears (open arrow) in the contrast-enhanced abdominal CT scan.

regular follow-up and image searches through annual CT scans are effective even after five years have passed since the initial treatment. Because our patient had a recurrence within the radiation field, repeated radiation therapy was difficult in view of the tolerable dose, so we implemented systemic chemotherapy and were able to control it.

In our case, the lymph node metastasis was not verified by pathological study. Current advances in the PET-CT can provide molecular information as well as morphological abnormalities. Tran et al. reported the PET and pathological correlation for lymph node metastases by $\mathrm{SCN}^{7}$. They concluded that the abnormal accumulation with PET has $100 \%$ accuracy even though the mass was not palpable. PET is currently used for determining the staging of cervical cancer ${ }^{11-14}$. Considering the clinical course and PET-CT findings, it is reasonable to make a diagnosis of the lymph node metastasis in the present case despite the lack of pathological proof.

In the past, the diagnosis of distant metastases before a recurrence of the clinical symptoms was extremely limited; however, diagnostic performance has improved with the appearance of the CT scans and MRI. With the appearance of PET-CT, it has become possible to diagnose the stage of metastases at an even earlier stage. As a result, it has become possible to create treatment plans for distant metastases early on. We anticipate that unfavorable prognoses for distant metastases patients with stage IVB had hitherto will improve from now on. Because PET is an expensive examination method, however, we think that research is necessary regarding the most appropriate examination timing and frequency.

\section{Conflict of Interest}

The authors declare that they have no conflict of interest.

\section{References}

1) Eifel PJ, Winter $K$, Morris $M$, Levenback $C$, Grigsby PW, Cooper J, Rotman M, Gershenson DM, Mutch DG. Pelvic irradiation with concurrent chemotherapy versus pelvic and para-aortic irradiation for high-risk cervical cancer: An update of Radiation Therapy Oncology group trial (RTOG) 90-01. J Clin Oncol 2004; 22: 872 880.

2) Rose PG, Ali S, Watkins E, Thigpen JT, Deppe G, Clarke-Peasons DL. Long-term follow-up of randomized trial comparing concurrent single agent cisplatin, cisplatin-based combination chemotherapy, or hydroxyurea during pelvic irradiation for locally advanced cervical cancer; a Gy- 
necologic Oncology Group Study. J Clin Oncol 2007; 25: 2804-2810.

3) Green JA, Kirwan JM, Tierney JF, Symonds P, Fresco, Collingwood LM, Williams CJ. Survival and recurrence after concomitant chemotherapy and radiotherapy for cancer of the uterine cervix: a systematic review and meta-analysis. Lancet 2001; 358: 781-786.

4) Kim K, Cho SY, Kim BJ, Kim MH, Choiv SC, Ryu SY. The type of metastasis is a prognostic factor in disseminated cervical cancer. J Gynecol Oncol 2010; 21: 186-190.

5) Gomi H, Shinozaki M, Okada Y, Abe T, Nakajima Y, Ohara T, Tozawa A, Suzuki N. Definitive radiotherapy for cervical cancer with paraaortic lymph nodes metastasis. J St. Marianna Univ 2015; 42: 265-273.

6) Kidd E, Siegel B, Dehdashti F, Rader J, Mutch D, Powell M, Grigsby P. Lymph Node Staging by Positron Emission Tomography in Cervical Cancer: Relationship to Prognosis. J Clin Oncol 2001; 19: 3745-3749.

7) Tran BN, Grigsby PW, Dehdashti F, Herzog TJ, Siegel BA. Occult supraclavicular lymph node metastasis identified by FDG-PET in patients with carcinoma of the uterine cervix. Gynecol Oncol 2003; 90: 572-576.

8) Qiu JT, Ho KC, Lai CH, Yen TC, Huang YT, Chao A, Chang TC. Supraclavicular lymph node metastases in cervical cancer. Eur J Gynaecol Oncol 2007; 28: 33-38.
9) Chao A, Ho K, Wang CC, Cheng HH, Lin G, Yen TC, Lai CH. Positron emission tomography in evaluation the feasibility of curative intent in cervical cancer patients with limited distant lymph node metastases. Gynecol Oncol 2008; 110: 172-178.

10) Kim JY, Kim JY, Kim JH, Yoon M, Kim J, Kim Y. Curative chemoradiotherapy in patients with stageIVB cervical cancer presenting with paraaortic and left supraclavicular lymph node metadtases. Int J Radiot Oncol Biol Phys 2012; 84: 741-747.

11) Rose PG, Adler LP, Rodriguez M, Faulhaber PF, Abdul-Karim FW, Miraldi F. Positron emission tomography for evaluating paraaortic nodal metastasis in locally advanced cervical cancer before surgical staging: A surgicopathologic study. J Clin Oncol 1999; 17: 41-45.

12) Sugawara $Y$, Eisbruch A, Kosuda S, Recker B, Kison P, Wahl R. Evaluation of FDG-PET in patients with cervical cancer. J Nucl Med 1999; 40: 1125-1131.

13) Grigsby PW, Siegel BA, and Dehdashti F. Lymph node staging by positron emission tomography in patients with carcinoma of the cervix. J Clin Oncol 2001: 19: 3745-3749.

14) Singh A, Grigsby P, Dehdashti F, Herzog TJ, Siegel B. FDG-PET lymph node staging and survival of patients with FIGO staging IIIb cervical carcinoma. Int J Radiot Oncol Biol Phys 2003; 56: 489-493. 